\title{
Predicción del índice de fermentación de cacao (Theobroma cacao $L$.) mediante análisis de imagen y redes neuronales
}

\author{
Mohamed Abderrahim \\ Universidad Carlos III de Madrid, Departamento de Ingeniería de Sistemas y Automática, \\ mohamed@ing.uc3m.es \\ Luis Condezo-Hoyos \\ Universidad Carlos III de Madrid, Departamento de Ingeniería de Sistemas y Automática, \\ lcondezotm@hotmail.com \\ Noemí León Roque \\ Universidad Nacional Pedro Ruíz Gallo, \\ Departamento de Ingeniería en Industrias Alimentarias, Lambayeque, Perú, \\ noemileon26@hotmail.com \\ Silvia M. Arribas \\ Universidad Autónoma de Madrid, Facultad de Medicina, silvia.arribas@uam.es
}

\section{RESUMEN}

En el presente estudio se desarrolló y validó un método de predicción del índice de fermentación de granos de cacao usando visión por computador y redes neuronales. El índice fermentación es un indicador de la calidad organoléptica de los granos procesados de cacao. Este control habitualmente se realiza en lotes de granos fermentados mediante la técnica Cut-Test que mide de manera subjetivamente el color. Se implementaron seis modelos de predicción empleando valores de RGB de la superficie y de la parte media de los granos de cacao $y$ de los extractos alcalinos. El índice de fermentación de cacao predichos mediante el modelo redes neuronales basado en los valores RGB de la superficie de los granos y los valores $\mathrm{R} / \mathrm{G}$ de los extractos se correlacionó positivamente con los valores experimentales $\left(\mathrm{R}^{2}=0.59\right)$. El análisis Bland-Altman y el análisis de regresión PassingBablok de validación confirmaron que el modelo puede ser usado para predecir el contenido de aminoácidos libres y consecuentemente el índice de fermentación de los granos de cacao. El modelo de predicción podría ser aplicado in situ en el procesamiento y el control de calidad del cacao mediante la implementación de apps para dispositivos móviles.

Palabras Clave: Cacao, indice de fermentación, visión por computador, redes neuronales

\section{INTRODUCCIÓN}

El cacao es uno de los cultivos más promisorio en los países en vías de desarrollo. La determinación del índice de fermentación es uno de los controles de calidad que permiten garantizar su calidad organoléptica como producto fermentado [4]. Este control habitualmente se realiza en lotes de granos fermentados mediante la técnica Cut-Test, que mide de manera subjetivamente el color, o a través de la medición de la razón de absorbancias de extractos preparados en metanol acidificado que al no ser lo suficientemente sensible no permite comparar distintos lotes de producto fermentado [1,7].

La química analítica basada en visión por computador (QA-VC) está enfocada en el análisis químico a partir de datos de color [2]. QA-VC es de enorme interés debido a que presenta una serie de ventajas entre las que resaltan su naturaleza no invasiva, simplicidad y portabilidad para aplicaciones in situ out-of-lab [2]. Sin embargo, QA-AC se ha empleado de manera limitada en la ciencia de alimentos [5,8].

El objetivo del presente estudio es predecir el índice de fermentación de granos de cacao completamente fermentados a partir de datos de color RGBabsorbancia y redes neuronales (RNs). Se validaron diferentes modelos RNs de predicción del índice de fermentación obtenidos a partir de datos de color de la superficie y de la parte media de los granos usando análisis de correlación lineal, Bland-Altman y análisis de correlación de Passing-Bablok. 


\section{MÉTODO PROPUESTO}

\subsection{Adquisición y análisis de imagen}

\subsubsection{Adquisición de imagen}

Las imágenes de 120 granos de cacao fermentados (superficie y medio) se adquirieron en un escáner estándar HP PSC 1510. El escáner se configuró con los siguientes parámetros: resolución $=1200$ ppi, profundidad de bits $=32$ y formato de imagen $=$ TIFF comprimido. En el presente estudio se emplearon dos variedades de cacao finos $\left(\mathrm{M}_{1} \mathrm{y} \mathrm{M}_{2}\right) \mathrm{y}$ uno criollo $\left(\mathrm{M}_{3}\right)$ procedentes de Piura y Cajamarca, Perú, respectivamente.

\subsubsection{Análisis de imagen}

El tratamiento y el análisis de imagen se hizo empleando Image Processing Toolbox de MATLAB. Los parámetros de color del método QA-VC se calcularon empleando el siguiente procedimiento:

\subsubsection{RGB de los granos de cacao}

La detección de los granos de cacao fermentados se realizó mediante un procedimiento MATLAB que se describe en el siguiente pseudocódigo:

\section{Pseudocódigo MATLAB}

Leer la imagen original

\% adquirida mediante cámara digital

$\mathrm{I} 2$ = grayscale (imagen original);

$\%$ Ajustar background

Background = imopen (I2, strel('disk',15));

$\mathrm{I} 3=\mathrm{I} 2$-Background;

$\%$ binarizar la imagen

level = graythresh (I3);

$\mathrm{bw}=\mathrm{im} 2 \mathrm{bw}(\mathrm{I} 3$, level $)$;

bw = bwareaopen(bw, 50);

imshow(bw, 'InitialMagnification', 33);

$\%$ Análisis de la imagen binarizada

bw = bwareaopen(IM2, 4000);

$\mathrm{cc}=$ bwconncomp $(\mathrm{bw}, 8)$;

grain $=$ false $(\operatorname{size}(b w))$;

grain (cc.PixelIdxList $\{1\})=$ true;

graindata $=$ regionprops $(\mathrm{cc}$, 'basic');

$\%$ Area del grano 1

Area = graindata(1).Area;

Rep = im2uint8(grain);

Repconv $=\operatorname{Rep} / 255$;

$\% R G B$ del grano 1

Rvalue = cacao $(:,:, 1) . *$ Repconv;

Gvalue $=$ cacao $(:,:, 2) . *$ Repconv;

Bvalue $=$ cacao $(:,:, 3) .{ }^{*}$ Repconv;

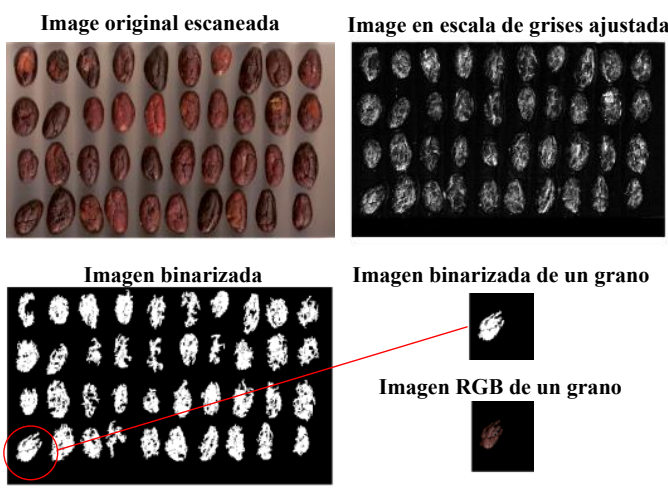

Figura 1. Salida del pseudocódigo empleado en el análisis RGB de los granos de cacao.

\subsubsection{Espectro de absorción de los extractos de grano de cacao}

Los granos fermentados (120) de cada una de la variedades de cacao se molieron individualmente en un molinillo de café (Selecline, France) usando 5 ciclos de $10 \mathrm{~s}$ para evitar que las muestras se calentaran durante el proceso. Los granos molidos se extrajeron con hidróxido de sodio $(0.28 \mathrm{~mol} / \mathrm{L})$ en un agitador de tubos (J.P. Selecta S.A, Barcelona, Spain) durante 5 minutos a temperatura ambiente. El extracto se centrifugo a $5000 \mathrm{rpm}$ por 5 minutos y espectro de absorción de 400-700 nm del líquido sobrenadante se midieron en un lector de placa robotizado (Synergy HT, Biotek, VT, USA). Asimismo, la placa se escaneo y los valores R G B de cada extracto se midieron empleado el siguiente pseudocódigo:

\section{Pseudocódigo MATLAB}

Leer la imagen original

$\%$ adquirida cámara digital

I2 = grayscale (imagen original);

$\%$ reducir pixeles en bordes

Calcular el tamaño de la imagen (I2)-> $>$ m n $]$

Calcular el paso entre pocillos -> p

$\%$ usando el tamaño y el número de pocillos por líneas (l) $y$ columnas (c) (sale $p=62$ en el ejemplo usado)

$\%$ Seleccionar un cuadrado de $27 \times 27$ pixeles por cada pocillo

For $\mathrm{j}=1: 1$

$$
\begin{aligned}
& \text { For } \mathrm{i}=1: \mathrm{c} \\
& \mathbf{P P}=\operatorname{original}(((\mathrm{i}-1) * \mathrm{p})+\mathrm{p} \\
& \mathrm{p})+\mathrm{p} 1+13, \mathrm{p} 2+((\mathrm{j}-1) * \mathrm{p})-1 \\
& :) ; \\
& \text { pocillos }\{(\mathrm{i}-1) * 6+\mathrm{j}\}=\mathbf{P P} \text {; }
\end{aligned}
$$$$
\mathbf{P P}=\operatorname{original}(((\mathrm{i}-1) * \mathrm{p})+\mathrm{p} 1-13:((\mathrm{i}-1) *
$$$$
\text { p) }+ \text { p } 1+13, p 2+\left((j-1)^{*} p\right)-13:\left((j-1)^{*} p\right)+p 2+13 \text {, }
$$

End

$\%$ p 1 y p2 son los índices del primer pocillo

$\%$ pocillos es un array de imágenes 


\subsection{Modelos de redes neuronales de predicción del índice de fermentación}

Seis modelos de redes neuronales Multilayer Perceptron de predicción del índice de fermentación de cacao se implementaron usando Neural Network Toolbox de MATLAB con los siguientes parámetros: 1) Número de neuronas en las capas ocultas = 3-10; 2) Redes para entrenar $=20$; 3) Redes para reentrenar $=5$ y 4 ) Funciones de activación identidad, logística, Tanh, exponencial y seno.

Los modelos de redes neuronales se construyeron empleando los siguientes datos de color de los granos fermentados:

I) RGB de la superficie y del medio.

II) RGB de la superficie, medio y extracto.

III) RGB de la superficie y espectro de absorción del extracto.

IV) RGB de la superficie-medio y espectro de absorción del extracto

V) RGB de la superficie y absorbancia del extracto a $420 \mathrm{~nm}$

VI) RGB de la superficie y R/G del extracto.

\subsection{Validación de los modelos de redes neuronales de predicción del índice de fermentación}

Para validar los modelos de redes neuronales se empleó como datos experimentales el contenido de aminoácidos libres de cada uno de los granos, el cual se midió mediante un método fluorimétrico de alta eficiencia [13]. El índice de fermentación (IF) experimental-predicho se calculó mediante la siguiente ecuación (1):

$$
I F=\frac{\sum_{i=1}^{i=n} \frac{A F_{f g i}}{A F_{u f g}}}{n}
$$

$A F$ es el contenido de aminoácidos libres en los granos fermentados $\left(A F_{f g}\right) i$ del lote y en los granos sin fermentar $\left(A F_{u f g}\right)$ y $n$ es el número de granos fermentados evaluados.

\section{RESULTADOS}

\subsection{Color de los granos de cacao fermentados}

La fermentación de los granos de cacao es una de las etapas críticas en la preparación de productos derivados con excelente calidad organoléptica ya que regula la formación de diferentes precursores del aroma [2]. Durante la fermentación del cacao se ha descrito que se producen significativos cambios del color [1]. Por esta razón, el score del Cut-Test es comúnmente empleado para describir el índice de fermentación de los granos de cacao [2,3]. Un procedimiento basado en la medida de la absorbancia de extractos metanólicos a $460 \mathrm{~nm}$ y $530 \mathrm{~nm}$ se ha propuesto como indicador de la fermentación del cacao $[4,7]$. Pese a que este indicador está asociado con los indicadores de valores de color L, a, b [4], no tiene la sensibilidad suficiente para comparar el índice de fermentación de muestras de cacao completamente fermentado en las que diferencias de color son mínimas (Figura 2).
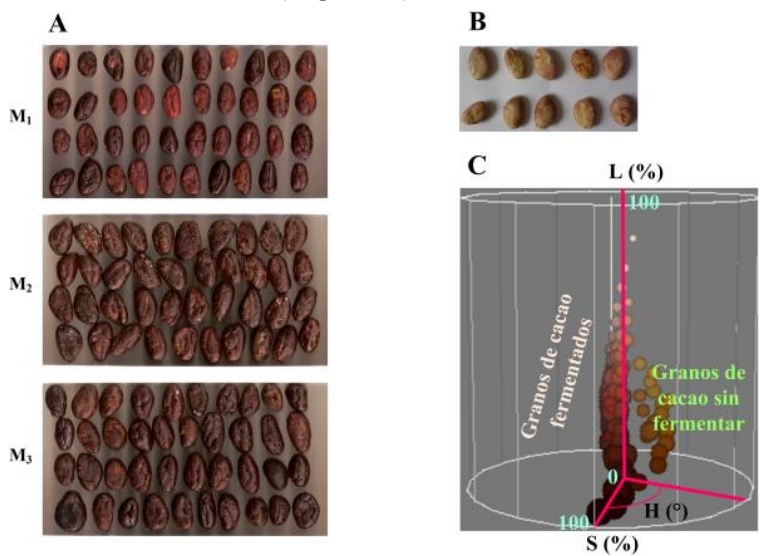

Figura 2. Imagen digital de granos de cacao fermentado (A) y sin fermentar (B) adquiridas con un escáner y un teléfono móvil, respectivamente. Valores de color de la superficie de los granos se representan en el espacio de color $\operatorname{HSL}(\mathrm{C}) . \mathrm{M}_{1}, \mathrm{M}_{2}$ and $\mathrm{M}_{3}$ son las muestras de cacao fermentado.

\subsection{Predicción del índice de fermentación de cacao usando modelos de redes neuronales}

El cambio de color que se produce durante la fermentación de los granos de cacao es un proceso bioquímico complejo que requiere de modelos no lineales complejos de predicción [4]. Los modelos de redes neuronales son no lineales y son de utilidad para predecir el comportamiento de sistemas complejos [7]. El índice de fermentación de granos de cacao predicho por los modelos de redes neuronales $\mathrm{V}$ y VI basado en datos de visión por computador implementados en el presente estudio mostraron la misma tendencia que los datos experimentales (Figura 3). El contenido de aminoácidos libres en los granos de cacao predichos mediante el modelo VI para las distintas fases de implementación de las redes neuronales son mostrados en la Figura 4. Estos datos se utilizaron para estimar el índice de fermentación de los granos de cacao empleando la ecuación (1). Aunque recientemente se ha implementado un método de predicción del nivel de fermentación de cacao basado en medidas de espectrofotometría infrarrojo cercano, su aplicabilidad en el procesamiento y control de calidad del cacao es limitado debido a que es una tecnología costosa [3]. Por lo tanto; el modelo VI de redes neuronales basado en medidas de color de la superficie de los granos y sus extractos podría ser una 
alternativa de bajo coste de aplicabilidad in situ outlab para el control de calidad del cacao fermentado.
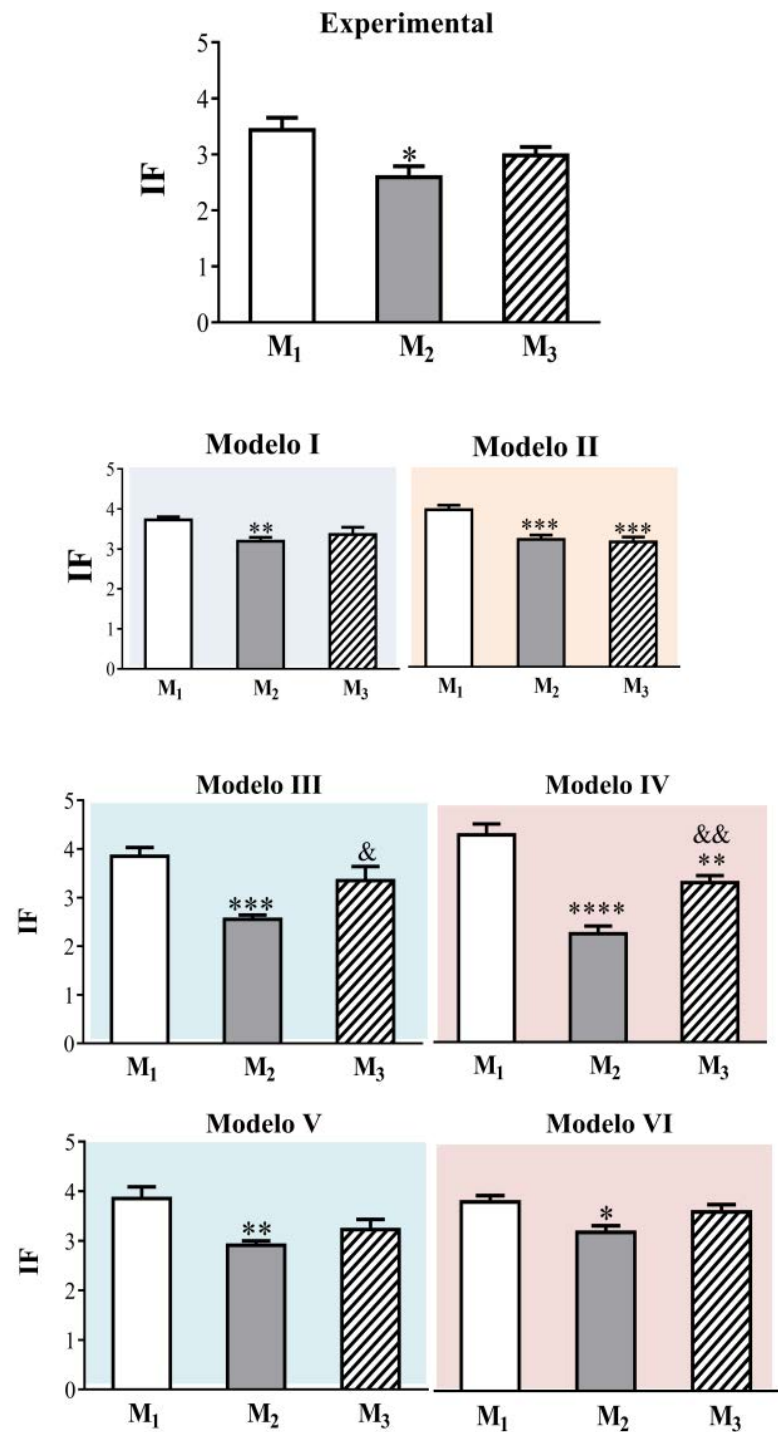

Figura 3. Índice de fermentación de granos de cacao experimental y predicho mediante los modelos de redes neuronales basado en datos de color. *, \& $\mathrm{p}<$ $0.05, * *, \& \& \mathrm{p}<0.01, * * \mathrm{p}<0.01 \mathrm{y} * * * \mathrm{p}<0.001$ denotan diferencias estadísticas.
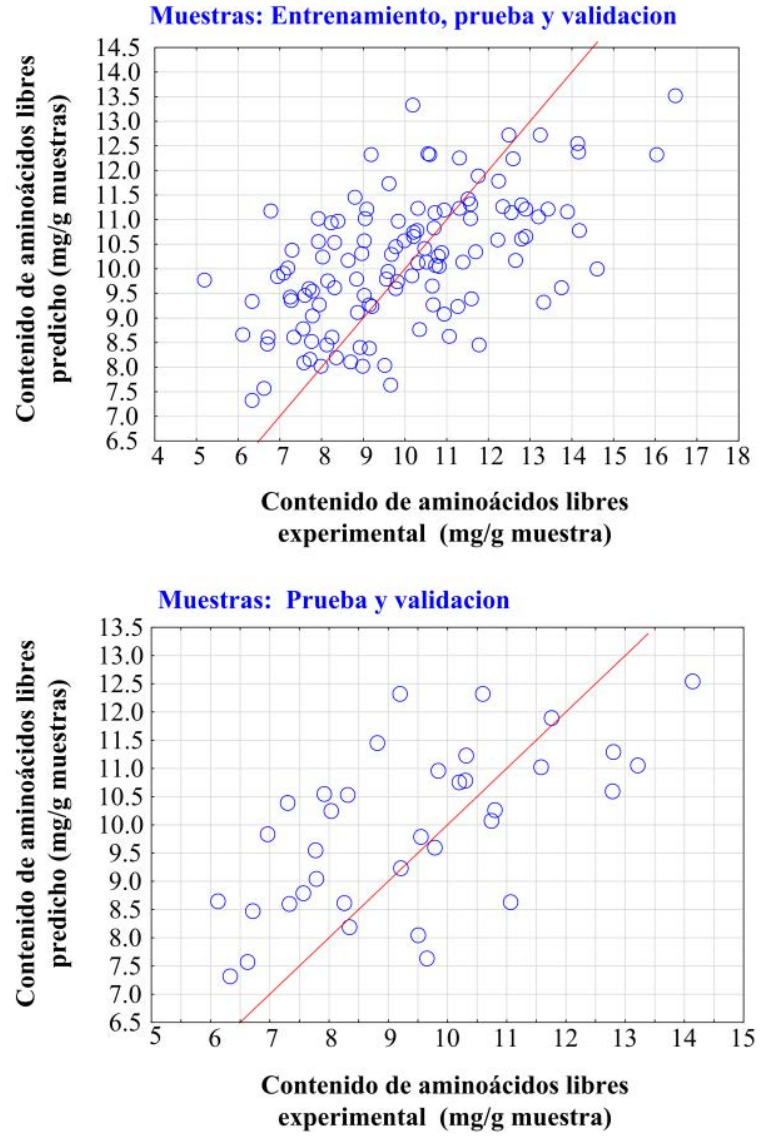

Figura 4. Contenido de aminoácidos libres de granos de cacao fermentados experimentales y predichos mediante el modelo VI de redes neuronales para las etapas de entrenamiento-prueba-validación, y prueba-validación

\subsection{Validación del modelo VI de redes neuronales de predicción del índice de fermentación}

Los índices de fermentación de cacao predichos mediante el modelo VI de redes neuronales se correlaciono positivamente con los valores experimentales $\left(\mathrm{R}^{2}=0.59\right)$ para los granos de cacao usados para la etapa de validación. El análisis BlandAltman confirmó que el modelo VI puede ser usado para predecir el contenido de aminoácidos libres consecuentemente el índice de fermentación de los granos de cacao. Finalmente, el análisis de regresión de Passing-Bablok demostró que no existe sesgo de la proporcionalidad entre el método fluorimétrico y el modelo VI de redes neuronales (IC 95\% de la pendiente $=0.9766$ to 4.6967 ), lo que significa que no muestran desviación significativa de la linealidad. Aunque, el intercepto si mostró sesgo (IC 95\% del intercepto $=-12.7368$ to -0.3333$)$ y es necesario incluir un valor de corrección en el modelo VI para predecir los aminoácidos libres y el índice de fermentación de los granos de cacao (Figura 5). 

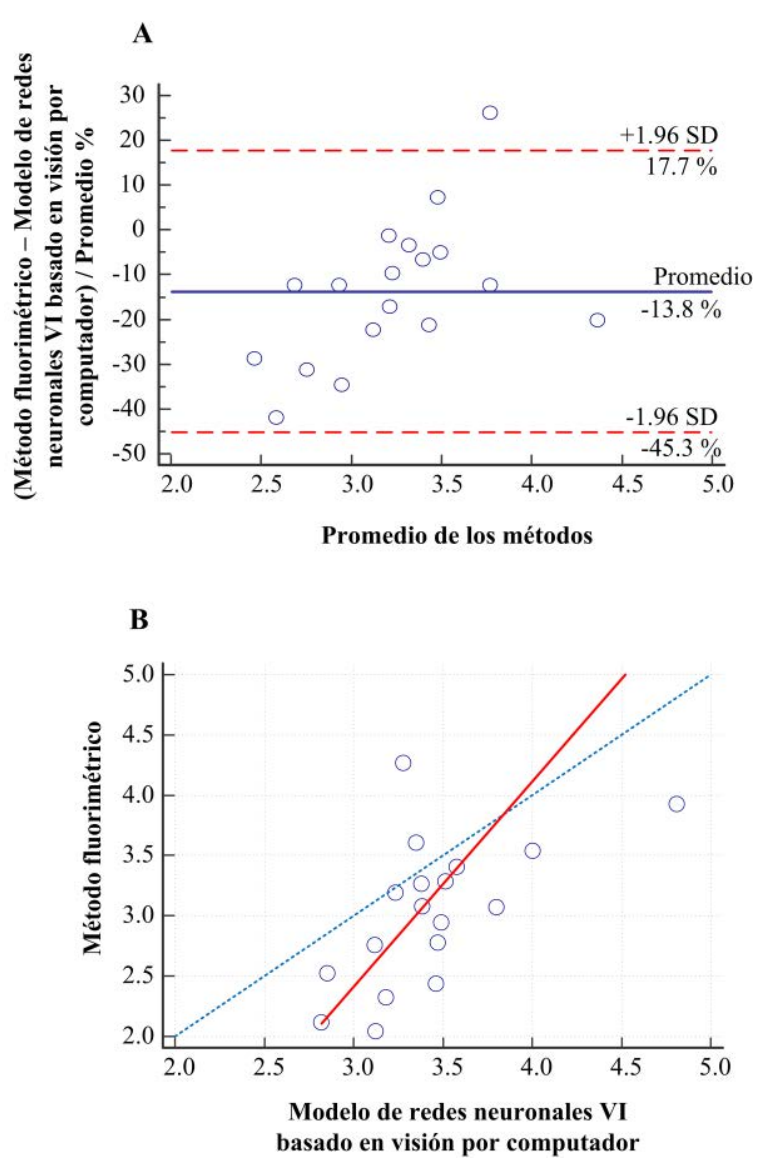

Figura 5. Validación del modelo VI de redes neuronales basado en medidas de color para predecir aminoácidos libres.

\section{CONCLUSIONES}

Se ha desarrollado y validado un modelo de redes neuronales basado en color para predecir el índice de fermentación de granos de cacao medido por el contenido de aminoácidos libres. El método analítico basado en visión por computador incluye como variables de predicción valores RGB de la superficie de los granos de cacao y de los extractos en hidróxido sodio. El método podría ser aplicado in situ en el procesamiento y el control de calidad del cacao mediante la implementación de apps para dispositivos móviles tales como smartphone y tabletas.

\section{REFERENCIAS}

[1] Amoa-Awua, W. (2015). Methods of Cocoa fermentation and Drying. In Cocoa and coffee fermentations, 1th ed., CRC Press - Taylor \& Francis Group, NY, USA.

[2] Capitán-Vallvey, L.F., López-Ruiz, N., Martínez-Olmos, A., Erenas, M.M., Palma, A.J. (2015). Recent developments in computer vision-based analytical chemistry: A tutorial review. Anal. Chim. Acta 899 23-56.

[3] Hue, C., Gunata, Z., Bergounhou, A., Assemat, S., Boulanger, R., Sauvage, F.X., Davrieux, F. (2014). Near infrared spectroscopy as a new tool to determine cocoa fermentation levels through ammonia nitrogen quantification. Food Chem. 148 240-245.

[4] Ilangantileke, S.G, Wahyudi, T., Bailon, M.G. (1991). Assessment methodology to predict quality of cocoa beans for export. J Food Qual. 14 481-496.

[5] Lu, H., Zheng, H. (2012). Fractal colour: A new approach for evaluation of acrylamide contents in biscuits. Food Chem. 134 2521-2525.

[6] Marini, F. (2009). Artificial neural networks in foodstuff analyses: Trends and perspectives A review. Anal. Chim. Acta 635 121-131.

[7] Misnawi, S., Jinap, B., Jamilah, S. Nazamid. (2003). Effects of incubation and polyphenol oxidase enrichment on colour, fermentation index, procyanidins and astringency of unfermented and partly fermented cocoa beans. Int. J. Food Sci. Tech 38 285-295.

[8] Pace, B., M. Cefola, M., Da Pelo, P., Renna, F., Attolico, G. (2014). Non-destructive evaluation of quality and ammonia content in whole and fresh-cut lettuce by computer vision system. Food Res. Int. 64 647-655. 